\title{
El tamaño y la duración previa predicen la curación en úlceras de pie diabético de origen neuropático
}

Risk Factors for Delayed Healing of Neuropathic Diabetic Foot Ulcers. Margolis D, Kantor J, Santana J et al. ARCH DERMATOL $2000 ; 136$ $1531-1555$

\section{Objetivo}

Estimar el efecto de varios factores de riesgo sobre la probabilidad de que las úlceras neuropáticas de pié diabético cicatricen en 20 semanas con tratamiento convencional (curación, debridamiento y descarga de presión del área afectada)

\section{Diseño}

Análisis combinado, tipo meta-análisis, con datos de pacientes individuales de las ramas de cuidado usual de cinco ensayos clínicos controlados aleatorizados.

\section{Lugar}

Universidad de Pennsylvania, Escuela de Medicina de Filadelfia, EE.UU.

\section{Pacientes}

Pacientes diabéticos $(n=586)$ con úlceras de pie neuropáticas que participaron como grupo control de cinco estudios clínicos randomizados. Todos recibieron tratamiento convencional.

\section{Evaluación de factores pronósticos}

Se evaluaron múltiples variables demográficas, tamaño de la úlcera, tiempo de evolución y hemoglobina gicosilada en los pacientes que curaron en 20 semanas vs. los que tardaron más de 20 semanas en curar. La regla de predicción final incluyó sólo las variables asociadas significativamente a resolución temprana.

\section{Medición de resultado principal}

Para calcular la magnitud de la asociación de cada factor de riesgo con la curación de las úlceras de los pacientes se utilizó un análisis multivariado.*

\section{Resultados}

El análisis de los datos detectó que las siguientes variables se asociaron a curación temprana de la úlcera neuropática en los pacientes con tratamiento convencional: lesiones $<2 \mathrm{~cm}$, tiempo de evolución $<6$ meses y no ser de raza blanca (Ver tabla). La edad, el nivel de hemoglobina glicosilada al comienzo del estudio y el sexo no se asociaron a la probabilidad de curar en 20 semanas.

Factores asociados a la resolución precoz de la úlceras neuropáticas en pacientes diabéticos

\begin{tabular}{l|c|c}
\hline Variable & Riesgo Relativo & IC $\mathbf{9 5 \%}$ \\
\hline Lesión $<2.0 \mathrm{~cm}$ & 0.67 & 0.55 a 0.81 \\
\hline Evolución previa <6 meses & 0.73 & 0.61 a 0.87 \\
\hline No ser de raza blanca & 0.64 & 0.43 a 0.96 \\
\hline
\end{tabular}

\section{Conclusiones}

En pacientes diabéticos con úlcera de pie neuropática la utilización de un tratamiento convencional estaría recomendada si la herida mide $<2 \mathrm{~cm}$ y tiene una evolución $<6$ meses. Las úlceras de mayor tamaño o de mayor tiempo de evolución deberían evaluarse en centros especializados.

\section{Comentario}

Las amputaciones en miembros inferiores son más frecuentes en pacientes diabéticos y más de 80.000 pac./año se amputan en EEUU. El $85 \%$ de las amputaciones en pacientes diabéticos son precedidas por una úlcera. ${ }^{\dagger}$ Además, el pie diabético es una de las complicaciones crónicas más costosas y que requiere más días de internación. ${ }^{2}$ En el $90 \%$ de los casos de úlcera plantar la neuropatia periférica es la principal causa. ${ }^{3-4}$ El tratamiento convencional de una úlcera diabética neuropática incluye: curación de la herida, debridamiento de los bordes y descarga de presiones de la zona ulcerada. Recientemente la Food and Drug Administration ha aprobado nuevas terapéuticas para el tratamiento de la úlcera diabética neuropática como el becaplermin (factor de crecimiento plaquetario ADN-recombinante ) y Apligraf (piel sintética), por lo cual sería importante determinar parámetros clínicos que orienten la elección correcta del tratamiento. ${ }^{5}$ Trabajos previos examinaron factores de riesgo asociados a cicatrización tardía en úlceras de distinto origen (neuropáticas, vasculares y mixtas). ${ }^{6}$ En el presente estudio se lograron identificar 3 factores de predicción de curación en un período igual o menor a 20 semanas con tratamiento convencional. Ellos fueron: el diámetro de la herida $(<2 \mathrm{~cm})$, el tiempo de evolución (< 6 meses ) y no ser de raza blanca. Si bien no existe ninguna evidencia biológica que justifique este último factor, los autores creen que hubo mayor adherencia para participar del estudio de los pacientes que no eran de raza blanca. No se halló asociación con la edad, el sexo ni el nivel de hemoglobina glicosilada al comenzar el estudio. Las críticas a este estudio serían: 1) no haber estudiado otros factores de riesgo como tipo de diabetes, tiempo de evolución de la misma, tabaquismo, comorbilidades y 2) no haber estudiado pacientes con macroangiopatía clínica.

En conclusión, dado que la úlcera diabética neuropática es una patología de tratamiento ambulatorio sería importante considerar el tamaño de la misma y el tiempo de evolución para elegir la opción terapéutica más adecuada.

Dra. Adriana Alvarez [ Servicio de Medicina Nuclear, Endocrinología y Metabolismo. Hospital Italiano de Buenos Aires ]

\footnotetext{
Referencias

1. Centers for Disease Control and Prevention. Diabetes -A Serious PublicHealth Problem. Atlanta, Ga: US Dept of Health and Human Services; $1988: 1-5$

2. Ramsey SD, Newton K, Blough D et al. Incidence, outcomes and costs of foot ulcers in patients with diabetes. Diabetes Care. 1999; $22: 382-387$.

3. Valway SÉ, Linkins RW, Godhes DM. Epidemiology of lower extremity amputations in the Indian Health Service, 1982-1987. Diabetes Care. 1993; 16:349-353.

4. Boulton AJM: Peripheral neuropathy and the diabetic. Foot 2:67-72,1992.

5. American Diabetes Association. Consensus Development Conference on Diabetes Wound Care: 7-8 april 1999, Boston, Massachusetts. Diabetes Care.1999; $22: 157-162$.

6. Rith -Najarian SJ, Stolusky T, Godhes DM. Identifying diabetic patients at high risk for lower-extremity amputation in a primary care setting : a prospective evaluation of simple screening criteria. Diabetes Care. 1992; 15:1386-1389.
} 